\title{
PARAMETRIZATIONS OF BOREL SETS WITH LARGE SECTIONS
}

\author{
A. MAITRA AND V. V. SRIVATSA
}

ABSTRaCt. The main result of this article unifies, extends, and simplifies the proofs of Borel parametrization theorems for Borel sets with large sections.

1. Introduction. Suppose $X$ is a separable metric space, $Y$ a Polish space, and $B$ a Borel subset of $X \times Y$ with uncountable vertical sections. A function $f: X \times 2^{\omega} \rightarrow X$ $\times Y$ is said to be a parametrization of $B$ if, for each $x \in X, f(x, \cdot)$ is a Borel isomorphism of $2^{\omega}$ and $\{x\} \times B_{x}$. If, moreover, $f$ is Borel measurable, we say that $f$ is a Borel parametrization of $B$.

Though Borel parametrizations were already implicit in the work of Purves [7], it was not until relatively recently that the first systematic study of the problem of finding Borel parametrizations of Borel sets with uncountable sections was undertaken. This was done by Mauldin in [4], which article is our point of departure. Roughly speaking, Mauldin proved, under the assumption that the horizontal axis $X$ is Polish, that the set $B$ admits a Borel parametrization provided that the vertical sections of $B$ are large. The precise sense in which the sections are large was expressed by Mauldin by assuming that either the vertical sections of $B$ have positive measure under a Borel measurable nonatomic transition function, or that the sections are nonmeager and $Y$ is perfect.

The aim of this article is to unify, extend, and simplify the proofs of these results of Mauldin. The extension is to the situation where the horizontal axis $X$ is separable metric. We achieve the unification by formulating the notion of largeness in terms of $\sigma$-ideals of the Borel $\sigma$-field of $Y$ satisfying a definability condition as in [3] and an extra condition. In [3] a similar unification was obtained with respect to measurable selectors for Borel sets with large sections. Indeed, the present article can be viewed as a continuation of [3].

The main result of the paper is

TheORem 1. Let $X$ be a separable metric space and $Y$ a Polish space. For each $x \in X$, let $\mathscr{J}_{x}$ be a $\sigma$-ideal of subsets of the Borel $\sigma$-field of $Y$ such that

(i) for each Borel set $C$ in $X \times Y$, the set $\left\{x \in X: C_{x} \notin \mathscr{J}_{x}\right\}$ is Borel in $X$, where $C_{x}=\{y \in Y:(x, y) \in C\}$, and

Received by the editors December 16, 1983 and, in revised form, April 9, 1984.

1980 Mathematics Subject Classification. Primary 54C65, 28B20, 28A05, 54H05; Secondary 03E15. $04 \mathrm{~A} 15$.

Key words and phrases. Borel parametrizations, Borel sets, measure, category. 
(ii) for each $x \in X, \mathscr{J}_{x}$ contains all singleton subsets of $Y$.

Let $B$ be a Borel set in $X \times Y$ such that $B_{x} \notin \mathscr{J}_{x}$ for each $x \in X$. Then there is $a$ one-one function $f: X \times 2^{\omega} \rightarrow X \times Y$ satisfying

(a) for each $x \in X, f(x, \cdot)$ is a Borel isomorphism of $2^{\omega}$ and $\{x\} \times B_{x}$, and

(b) $f$ and $f^{-1}$ are Borel measurable.

Condition (i) above ensures the existence of a Borel measurable selector for $B$; see [3]. Condition (ii) unifies the measure-theoretic condition in Mauldin's measure result that the transition function be nonatomic with the topological requirement in his category result that the space $Y$ be perfect.

The main theorem will be proved in $\S 3$. In $\$ 2$ we explain the notation to be used. $\S 4$ will discuss special cases of our theorem. In $\$ 5$ we present necessary and sufficient conditions for a Borel set to admit a Borel measurable parametrization and for a Borel set to admit a Borel measurable selector.

2. Notation. We denote the set of natural numbers by $\omega$. Finite sequences of natural numbers will be identified with their sequence numbers (see [6]). The set of sequence numbers is denoted by Seq. We denote by $\mathrm{Seq}_{2}$ the set of sequence numbers of finite sequences of 0 's and 1's. If $n \in \omega$, denote by $\langle n\rangle$ the sequence number of the one-element sequence $(n)$. For $s, t \in$ Seq, we write $s \subseteq t$ to denote that the finite sequence coded by $s$ is an initial segment of the finite sequence coded by $t$; write $s \subsetneq t$ if the sequence coded by $s$ is a proper initial segment of the sequence coded by $t$. If $s, t \in \mathrm{Seq}, s * t$ denotes the catenation of the sequence coded by $s$ followed by the sequence coded by $t$. The length of the sequence coded by $s \in$ Seq is denoted by $\operatorname{lh}(s)$.

The set of infinite sequences of natural numbers (0's and 1's) is denoted by $\omega^{\omega}\left(2^{\omega}\right)$. If $\alpha \in \omega^{\omega}, n \in \omega$, then $\alpha(n)$ denotes the $n$th coordinate of $\alpha$, and $\bar{\alpha}(n)$ denotes the sequence number of the finite sequence $(\alpha(0), \alpha(1), \ldots, \alpha(n-1))$. For $s \in$ Seq, set $N(s)=\left\{\alpha \in \omega^{\omega}: \bar{\alpha}(\operatorname{lh}(s))=s\right\}$. The sets $N(s), s \in$ Seq, form a base for a topology on $\omega^{\omega}$. Endowed with this topology $\omega^{\omega}$ becomes a Polish space which is homeomorphic to the space of irrationals. The set $2^{\omega}$ is topologized in a similar fashion so that it becomes a homeomorph of the Cantor set.

If $X$ is a separable metric space, $\mathfrak{B}(X)$ will denote the Borel $\sigma$-field of $X$.

Unexplained notation and terminology are from [6].

3. Proofs. We begin with the statement of a result (Lemma 3.4) from [8], to which the reader is referred for a proof.

Lemma 1. Let $X$ be a separable metric space. Suppose $h: X \times 2^{\omega} \rightarrow 2^{\omega}$ is a Borel measurable function such that for each $x \times X, h(x, \cdot)$ is a homeomorphism. Let $\hat{h}(x, \alpha)=(x, h(x, \alpha)), x \in X, \alpha \in 2^{\omega}$. Then, for each Borel set $E$ in $X \times 2^{\omega}, \hat{h}(E)$ is Borel in $X \times 2^{\omega}$.

The proof of the theorem is executed by reducing it to a special case. We consider the special case first. 
Lemma 2. Suppose $X$ is a separable metric space and let $A$ be a Borel subset of $X \times \omega^{\omega}$ with closed vertical sections. For each $x \in X$, let $\mathscr{I}_{x}$ be a $\sigma$-ideal of the Borel $\sigma$-field of $\omega^{\omega}$ such that

(i) for each $x \in X, A_{x} \notin \mathscr{I}_{x}$,

(ii) for each $(x, \alpha) \in A,\{\alpha\} \in \mathscr{I}_{x}$, and

(iii) for each open set $V$ in $\omega^{\omega}$, the set $\left\{x \in X: A_{x} \cap V \notin \mathscr{I}_{x}\right\}$ is a Borel set in $X$.

Then there is a Borel parametrization $g$ of $A$ such that $g^{-1}$ is Borel measurable.

Proof. Let $T(s)=\left\{x \in X: A_{x} \cap N(s) \in \mathscr{I}_{x}\right\}, s \in$ Seq. By hypothesis, $T(s)$ is Borel in $X$. Set

$$
M=A \backslash \bigcup\{T(s) \times N(s): s \in \mathrm{Seq}\} .
$$

Plainly $M$ is Borel in $X \times \omega^{\omega}, M_{x} \notin \mathscr{I}_{x}$ for each $x \in X, M_{x}$ is closed in $\omega^{\omega}$ for each $x \in X$, and for any $t \in$ Seq,

$$
M_{x} \cap N(t) \neq \varnothing \leftrightarrow A_{x} \cap N(t) \notin \mathscr{I}_{x} .
$$

It follows that $\left\{x \in X: M_{x} \cap N(t) \neq \varnothing\right\}$ is Borel in $X$ for each $t \in$ Seq. Since $\mathscr{I}_{x}$ contains singleton subsets of $A_{x}$, if $M_{x} \cap N(t) \neq \varnothing$, then $M_{x} \cap N(t)$ is uncountable. So $M_{x}$ is perfect for each $x \in X$.

Next we define a function $\varphi: X \times \mathrm{Seq}_{2} \rightarrow$ Seq satisfying the following conditions:

(a) for each $u \in \operatorname{Seq}_{2}, \varphi(\cdot, u)$ is Borel measurable on $X$,

(b) $u, v \in \operatorname{Seq}_{2} \& u \subseteq v \rightarrow \varphi(x, u) \subseteq \varphi(x, v)$,

(c) $u, v \in \operatorname{Seq}_{2} \& \operatorname{lh}(u)=\operatorname{lh}(v) \& u \neq v \rightarrow N(\varphi(x, u)) \cap N(\varphi(x, v))=\varnothing$,

(d) for each $u \in \operatorname{Seq}_{2}, \operatorname{lh}(\varphi(x, u)) \geqslant \operatorname{lh}(u)$, and

(e) $M_{x} \cap N(\varphi(x, u)) \neq \varnothing$ for each $u \in \operatorname{Seq}_{2}$.

The function $\varphi$ is defined by induction on $\operatorname{lh}(u)$. Set $\varphi(x, 1) \equiv 1$. (Recall that 1 is the sequence number of the empty sequence.) Suppose now that $\varphi(x, v)$ has been defined for all $v \in \operatorname{Seq}_{2}$ such that $\operatorname{lh}(v) \leqslant k$. Fix $u \in \operatorname{Seq}_{2}$ such that $\operatorname{lh}(u)=k$. We have to define $\varphi(x, u *\langle 0\rangle)$ and $\varphi(x, u *\langle 1\rangle)$. Set

$$
\begin{array}{r}
\varphi(x, u *\langle 0\rangle)=\mu t\left[\operatorname{Seq}(t) \& \varphi(x, u) \varsubsetneqq t \& M_{x} \cap N(t) \neq \varnothing\right. \\
\&(\exists s)\left(\operatorname{Seq}(s) \& \varphi(x, u) \varsubsetneqq s \& M_{x} \cap N(s) \neq \varnothing\right. \\
\& N(s) \cap N(t)=\varnothing)]
\end{array}
$$

and

$$
\begin{aligned}
\varphi(x, u *\langle 1\rangle)=\mu s[\operatorname{Seq}(s) \& \varphi(x, u) & \subsetneq s \& M_{x} \cap N(s) \neq \varnothing \\
& \& N(\varphi(x, u *\langle 0\rangle)) \cap N(s)=\varnothing] .
\end{aligned}
$$

Since, by the induction hypothesis, $M_{x} \cap N(\varphi(x, u)) \neq \varnothing$, and since $M_{x}$ is perfect, the functions $\varphi(x, u *\langle 0\rangle)$ and $\varphi(x, u *\langle 1\rangle)$ are well defined. Borel measurability of these functions follows from the condition that for each $s \in$ Seq the set $\{x \in X$ : $\left.M_{x} \cap N(s) \neq \varnothing\right\}$ is Borel in $X$ and the induction hypothesis.

The function $\varphi$ enables us to define a parametrization into $A$. Indeed, define $h$ : $X \times 2^{\omega} \rightarrow \omega^{\omega}$ by setting $h(x, \alpha)$ to be the unique element of $\bigcap\{N(\varphi(x, \bar{\alpha}(n))$ : $n \in \omega\}$. It is a straightforward matter to check that for each $x \in X, h(x, \cdot)$ is a homeomorphism of $2^{\omega}$ into $A_{x}$. Moreover, using the fact that the function $\varphi(x, u)$ is 
Borel measurable in $x$, one verifies easily that for each fixed $\alpha \in 2^{\omega}, \varphi(\cdot, \alpha)$ is a Borel measurable function on $X$. It now follows from a classical result (see [2]) that $h$ is Borel measurable.

To complete the proof we have to define a parametrization onto $A$. This is done by a Cantor-Bernstein type argument as in [4]. The details are as follows. First imbed $\omega^{\omega}$ as a $G_{\delta}$ in $2^{\omega}$, so we can think of $A$ as a Borel subset of $X \times 2^{\omega}$. Define $\hat{h}$ : $X \times 2^{\omega} \rightarrow X \times 2^{\omega}$ by setting $\hat{h}(x, \alpha)=(x, h(x, \alpha))$. Plainly $\hat{h}$ is Borel measurable, and, by Lemma $1, \hat{h}(E)$ is Borel in $X \times 2^{\omega}$ whenever $E$ is Borel in $X \times 2^{\omega}$. Define sets $S_{n}$ inductively as follows:

$$
\begin{aligned}
S_{0} & =\left(X \times 2^{\omega}\right) \backslash A, \\
S_{n+1} & =\left(X \times 2^{\omega}\right) \backslash\left(A \backslash \hat{h}\left(S_{n}\right)\right), \quad n \in \omega .
\end{aligned}
$$

Let $S^{*}=\cup_{n \in \omega} S_{n}$ and define $g: X \times 2^{\omega} \rightarrow X \times 2^{\omega}$ by

$$
\begin{array}{rlrl}
g(x, \alpha) & =\hat{h}(x, \alpha) & & \text { if }(x, \alpha) \in S^{*}, \\
=(x, \alpha) & \text { if }(x, \alpha) \in\left(X \times 2^{\omega}\right) \backslash S^{*} .
\end{array}
$$

Since the sets $S_{n}$ are Borel in $X \times 2^{\omega}$, so is $S^{*}$. It now follows easily that $g$ is a Borel parametrization of $A$ and $g^{-1}$ is Borel measurable. This completes the proof.

We now turn to the

Proof of Theorem 1. Let $\tilde{X}$ be the metric completion of $X$. Choose a Borel subset $\tilde{B}$ of $\tilde{X} \dot{\times} Y$ such that $B=\tilde{B} \cap(X \times Y)$. By a classical result (see [1]) there is a closed subset $D$ of $\omega^{\omega}$ and a one-one continuous function $h$ on $D$ onto $\tilde{B}$ which takes Borel sets in $D$ to Borel sets in $\tilde{B}$. Let $A=\left\{(x, \alpha) \in X \times D: \pi_{1} \circ h(\alpha)=x\right\}$, where $\pi_{1}$ is projection to the first coordinate. Observe that $A$ is a closed subset of $X \times \omega^{\omega}$ and $h$ maps $A_{x}$ in a one-one manner onto $\{x\} \times B_{x}$ for each $x \in X$.

Let $\mathscr{I}_{x}=\left\{h^{-1}(\{x\} \times E): E \subseteq B_{x} \& E \in \mathscr{J}_{x}\right\}, x \in X$. Then, for each $x \in X, \mathscr{I}_{x}$ is a $\sigma$-ideal of $\mathfrak{B}\left(\omega^{\omega}\right)$, and, since $\mathscr{J}_{x}$ contains all singleton subsets of $Y, \mathscr{I}_{x}$ contains all singleton subsets of $A_{x}$. Moreover, $A_{x} \notin \mathscr{I}_{x}$ as $B_{x} \notin \mathscr{J}_{x}$. Finally, if $V$ is an open set in $\omega^{\omega}$, then

$$
\left\{x \in X: A_{x} \cap V \notin \mathscr{I}_{x}\right\}=\left\{x \in X:(h(D \cap V) \cap(X \times Y))_{x} \notin \mathscr{J}_{x}\right\} .
$$

By condition (i) of the theorem and the bimeasurability of $h$, the set on the right side is Borel in $X$; hence, so is the set on the left. Consequently, $A$ and $\mathscr{I}_{x}, x \in X$, satisfy the hypotheses of Lemma 2, which yields a Borel measurable parametrization $g$ of $A$ such that $g^{-1}$ is Borel measurable. Let $f=h \circ \pi_{2} \circ g$, where $\pi_{2}$ is projection to the second coordinate. It is now easy to verify that $f$ is a Borel parametrization of $B$ such that $f^{-1}$ is Borel measurable. This completes the proof of the theorem.

REMARK. The idea of reducing the problem of finding a parametrization for a Borel set to that of finding one for a Borel set with closed vertical sections can also be used to give a very simple proof of the selection theorem in [3]. Indeed, we can proceed as in the proof of the theorem of the present article, getting a one-one continuous function $h$ on a closed subset $D$ of $\omega^{\omega}$, a closed set $A$ in $X \times \omega^{\omega}$, and the $\sigma$-ideals $\mathscr{I}_{x}$ of $\mathfrak{B}\left(\omega^{\omega}\right), x \in X$. The only difference now is that $\mathscr{I}_{x}$ may contain some singleton subsets of $A_{x}$, since, as per the hypotheses of the selection theorem of [3], 
$\mathscr{J}_{x}$ may contain singleton subsets of $Y$. Next we construct the set $M$ from $A$ as in the proof of Lemma 2. The set $M$ will have the same properties as the $M$ of Lemma 2, except that $M_{x}$ may not be perfect. But the last circumstance is irrelevant. The properties that $M$ enjoys are already sufficient for the Kuratowski-Ryll-Nardzewski selection theorem (see [2]) to be applicable, giving us a Borel measurable selector $g$ : $X \rightarrow \omega^{\omega}$ for the set $M$. Setting $f=\pi_{2} \circ h \circ g$, we get a Borel measurable selector for $B$.

4. Special cases. In this final section we deduce several known parametrization theorems from the main result of this paper.

Let $X$, then, be a separable metric space, $Y$ a Polish space, and $B$ a Borel set in $X \times Y$.

$1^{\circ}$. We consider Mauldin's measure result first. Let $Q(x, E), x \in X, E \in \mathfrak{B}(Y)$, be a Borel measurable transition function such that $Q(x, \cdot)$ is a nonatomic probability measure for each $x \in X$. We assume that $Q\left(x, B_{x}\right)>0, x \in X$. Let

$$
\mathscr{J}_{x}=\{E \in \mathfrak{B}(Y): Q(x, E)=0\}, \quad x \in X .
$$

It is an easy fact of measure theory that the $\sigma$-ideals $\mathscr{J}_{x}, x \in X$, satisfy the definability hypothesis of our theorem. Since each $Q(x, \cdot)$ is nonatomic, $\mathscr{J}_{x}$ contains all singleton subsets of $Y$, so that condition (ii) of our theorem is also satisfied. Mauldin's measure result is clearly implied by our theorem.

$2^{\circ}$. We turn now to Mauldin's category result, where we assume that $B_{x}$ is nonmeager in $Y$ for each $x \in X$ and $Y$ is perfect. We define

$$
\mathscr{J}_{x}=\{E \in \mathfrak{B}(Y): E \text { is meager in } Y\}, \quad x \in X .
$$

Again it is well known that the $\sigma$-ideals $\mathscr{J}_{x}, x \in X$, satisfy condition (i) in our theorem (see [9]). Condition (ii) is satisfied as $Y$ is perfect. Mauldin's category result now falls out of our theorem.

$3^{\circ}$. For our third example we make the following assumptions on $B$ : (a) the multifunction $x \rightarrow B_{x}$ is Borel measurable, i.e. the set $\left\{x \in X: B_{x} \cap V \neq \varnothing\right\}$ is Borel in $X$ for each open set $V$ in $Y$, (b) $B_{x}$ is nonmeager in $\operatorname{cl}\left(B_{x}\right)$ for each $x \in X$, and (c) $\operatorname{cl}\left(B_{x}\right)$ is perfect for each $x \in X$, where cl denotes the closure operator in $Y$. For each $x \in X$, we define $\mathscr{J}_{x}$ to be the $\sigma$-ideal of $\mathfrak{B}(Y)$ generated by the family

$$
\left\{E \subseteq \operatorname{cl}\left(B_{x}\right): E \in \mathfrak{B}(Y) \& \text { meager in } \operatorname{cl}\left(B_{x}\right)\right\} \cup\left\{Y \backslash \operatorname{cl}\left(B_{x}\right)\right\} \text {. }
$$

Using the methods of [9], one easily proves that the $\sigma$-ideals $\mathscr{J}_{x}, x \in X$, satisfy condition (i) of our theorem. Condition (ii) holds because of (c). It now follows from our theorem that there is a Borel parametrization of $B$, a result which was proved for analytic $X$ by Mauldin and Srivastava [5].

5. Necessary and sufficient conditions for existence of parametrizations and selectors. It was observed in the previous section that Mauldin's measure-theoretic result was a special case of our main theorem. But, as pointed out by the referee of this paper, Mauldin's hypotheses are, in fact, equivalent to the existence of a Borel measurable parametrization. To complete this circle of ideas, we incorporate this observation in the following theorem which gives necessary and sufficient conditions for a Borel set to admit a Borel measurable parametrization. 
Theorem 2. Let $X$ be a separable metric space, $Y$ a Polish space and, $B$ a Borel subset of $X \times Y$. Then the following conditions on the Borel set $B$ are equivalent:

(I) There is a nonatomic Borel measurable transition function $Q(x, E), x \in X$, $E \in \mathfrak{B}(Y)$, such that $Q\left(x, B_{x}\right)>0$ for each $x \in X$.

(II) There exist $\sigma$-ideals $\mathscr{J}_{x}, x \in X$, of $\mathfrak{B}(Y)$ satisfying conditions (i) and (ii) of Theorem 1 and such that $B_{x} \notin \mathscr{J}_{x}$ for each $x \in X$.

(III) There is a one-one function $f: X \times 2^{\omega} \rightarrow X \times Y$ satisfying (a) and (b) of Theorem 1.

The implication (I) $\rightarrow$ (II) is trivial, while (II) $\rightarrow$ (III) is just our main theorem. Finally, to prove (III) $\rightarrow$ (I), one fixes a nonatomic probability measure $\mu$ on the Borel subsets of $2^{\omega}$ and defines $Q(x, \cdot)=\mu f(x, \cdot)^{-1}$. It is easy to see that $Q$ satisfies (I). The equivalence (I) $\leftrightarrow$ (III) was observed by Mauldin in [4] for Polish $X$.

In view of the Remark at the end of $\$ 3$, one can formulate entirely analogous necessary and sufficient conditions for a Borel set $B \subseteq X \times Y$ to admit a Borel selector as follows.

Theorem 3. Let $X$ be a separable metric space, $Y$ a Polish space, and $B$ a Borel subset of $X \times Y$. Then the following conditions on the Borel set $B$ are equivalent:

(I) There is a Borel measurable transition function $Q(x, E), x \in X, E \in \mathfrak{B}(Y)$, such that $Q\left(x, B_{x}\right)>0$ for each $x \in X$.

(II) There exist $\sigma$-ideals $\mathscr{J}_{x}, x \in X$, of $\mathfrak{B}(Y)$ satisfying condition (i) of Theorem 1 and such that $B_{x} \notin \mathscr{J}_{x}$ for each $x \in X$.

(III) There is a function $f: X \rightarrow Y$ such that $(x, f(x)) \in B$ for each $x \in X$.

\section{REFERENCES}

1. K. Kuratowski, Topologv, Vol. 1, 5th ed., Academic Press, New York, 1966.

2. K. Kuratowski and C. Ryll-Nardzewski, A general theorem on selectors, Bull. Acad. Polon. Sci. Ser. Math. Astronom. Phys. 13 (1965), 397-403.

3. A. Maitra, Selectors for Borel sets with large sections, Proc. Amer. Math. Soc. 89 (1983), 705-708.

4. R. D. Mauldin, Borel parametrizations, Trans. Amer. Math. Soc. 250 (1979), 223-234.

5. R. D. Mauldin and S. M. Srivastava, Paramétrisations des ensembles coanalvtiques à coupes relativement non maigres, C. R. Acad. Sci. Paris Sér. A 292 (1981), 743-745.

6. Y. N. Moschovakis, Descriptive set theory, North-Holland, Amsterdam, 1980.

7. R. Purves, Bimeasurable functions, Fund. Math. 58 (1966), 149-157.

8. V. V. Srivatsa, Measurable parametrizations of sets in product spaces, Trans. Amer. Math. Soc. 270 (1982), 537-556.

9. R. L. Vaught, Invariant sets in logic and topologv, Fund. Math. 82 (1974), 269-294.

Indian Statistical. Institute, 203 B. T. Road, Calcutta 700 035, India 